\title{
Dynamic properties and optical phase conjugation of two-photon pumped ultrashort blue stimulated emission in a chromophore solution
}

\author{
Guang S. He, ${ }^{1, *}$ Hai-Yan Qin, ${ }^{1,3,4}$ Qingdong Zheng, ${ }^{1}$ Paras N. Prasad, ${ }^{1}$ Steffen Jockusch, ${ }^{2}$ Nicholas J. Turro, ${ }^{2}$ Marlin Halim, ${ }^{2}$ \\ Dalibor Sames, ${ }^{2}$ Hans Ågren, ${ }^{3}$ and Sailing $\mathrm{He}^{4}$ \\ ${ }^{1}$ Institute for Lasers, Photonics and Biophotonics, Department of Chemistry, State University of New York at Buffalo, \\ Buffalo, New York 14260-3000, USA \\ ${ }^{2}$ Department of Chemistry, Columbia University, 3000 Broadway, New York, New York 10027, USA \\ ${ }^{3}$ Department of Theoretical Chemistry, Royal Institute of Technology, S-106 91 Stockholm, Sweden \\ ${ }^{4}$ Centre for Optical and Electromagnetic Research, Zhejiang University, Hangzhou 310058, China \\ (Received 23 October 2007; revised manuscript received 15 November 2007; published 23 January 2008)
}

The dynamic properties of two-photon pumped blue lasing $(\sim 470 \mathrm{~nm})$ in the solution of an organic chromophore [2-acetyl-6-(dimethylamino)naphthalene], excited by $\sim 160-\mathrm{fs}$ laser pulses at $\sim 775 \mathrm{~nm}$, have been studied. Both the forward and backward stimulated emission are enhanced by feedback from the reflection at the two optical windows of the solution filled cuvette. Under current experimental conditions, the lasing wavelengths in the forward and backward directions were almost the same, but both blueshifted compared to the fluorescence peak wavelength of the sample solution. The temporal behavior of the lasing output was recorded by a high-speed streak camera system. The multipulse structure and spectral properties of the output lasing are semiquantitatively explained. In addition, excellent optical phase-conjugation properties of the backward stimulated emission were observed; the aberration influences from an aberrator on the backward lasing beam were automatically removed.

DOI: 10.1103/PhysRevA.77.013824

PACS number(s): 42.65.Sf, 32.80.Wr, 42.65.Re, 42.65.Hw

\section{INTRODUCTION}

Multiphoton pumped (MPP) up-conversion lasing in organic gain media has attracted considerable attention in the past decade [1-14]. One unique feature of multiphoton pumped stimulated emission is that the pump wavelength can be chosen in a suitable infrared (IR) range and the stimulated emission generated can be in the visible range. Compared to other existing frequency up-conversion techniques (such as second- or third-harmonic generation), MPP lasing exhibits the advantage of easy tunability and no phasematching requirement. For some applications, such as optical data storage and photolithography, a shorter lasing wavelength is more desirable. So far, most reported MPP lasing wavelengths were in the 490-620-nm spectral range.

In this work, we report experimental studies of twophoton pumped (2PP) blue lasing at a wavelength of $\sim 470 \mathrm{~nm}$ from a chromophore solution, excited by femtosecond IR laser pulses. One feature of this work is to investigate the role of feedback from the reflection at the two windows of the sample cuvette. It is found that the feedback can enhance the lasing output and affect the lasing dynamic behavior. Another feature is that the lasing wavelengths are shorter than the peak fluorescence wavelength. This is explained by the transient behavior of the peak gain position.

In addition, experimental results of optical phaseconjugation property for the backward lasing beam are also presented. This property indicates that a good optical quality lasing beam can be obtained from a poor quality lasing medium or even after passing through a highly aberrated propagation medium.

\footnotetext{
*gshe@acsu.buffalo.edu
}

\section{OPTICAL PROPERTIES OF A LASING MEDIUM}

The 2PP lasing medium in this study is an organic chromophore, 2-acetyl-6-(dimethylamino)naphthalene (ADN) in dimethyl sulfoxide (DMSO) solution. The chemical structure of ADN is shown at the top of Fig. 1(a). This chromophore was originally designed as fluorogenic probe for biological systems using two-photon excitation [15], and the synthesis of ADN was described previously [16].

The measured linear absorption spectra of a 1-mm-thick dilute solution sample of ADN and pure solvent (DMSO) of the same path length are shown in Fig. 1(a), respectively, where ADN shows a linear absorption peak at $\sim 360 \mathrm{~nm}$ with a bandwidth of $\sim 70 \mathrm{~nm}$. Shown in Fig. 1(b) are the transmission spectra of a 1-cm-path-length solution sample of $0.05 M$ concentration and a pure DMSO sample of the same path length. From Fig. 1(b) one can see that there is negligible linear absorption for the solute in the entire spectral range from 600 to $1500 \mathrm{~nm}$, although there are several discrete IR absorption bands attributed to the solvent.

A Ti:sapphire laser oscillator-amplifier system (CPA-2010 from Clark-MXR) was utilized as a two-photon excitation source, which generated pulses at $775 \mathrm{~nm}$ of $160 \mathrm{fs}$ pulse length. This nearly linearly polarized laser beam has a beam size of $\sim 4 \mathrm{~mm}$, a divergence angle of $0.22 \mathrm{mrad}$, and a repetition rate of $1 \mathrm{kHz}$.

The one-photon induced fluorescence spectrum from a 1-mm ADN solution sample of low $(0.0005 M)$ concentration is shown in Fig. 2 (solid line), which was excited at $388 \mathrm{~nm}$ by using second-harmonic generation of the 775-nm laser beam. The peak fluorescence wavelength is located at $\sim 475 \mathrm{~nm}$, and the fluorescence spectral bandwidth (full width at half maximum) is $\sim 70 \mathrm{~nm}$. With excitation at $775 \mathrm{~nm}$, the two-photon absorption (2PA) induced fluores- 

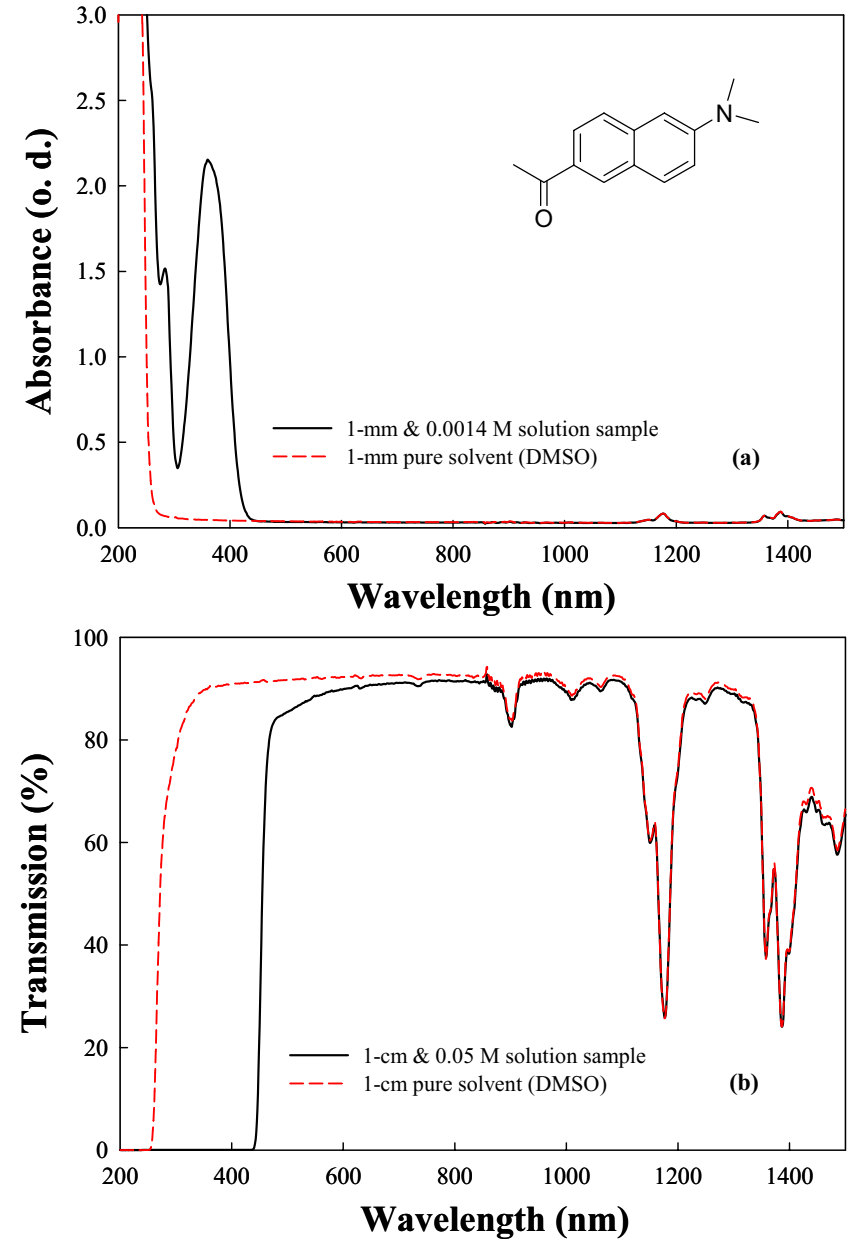

FIG. 1. (Color online) (a) The linear absorption spectra of a 1-mm diluted ADN solution in DMSO (solid line) and a 1-mm pure DMSO sample (dashed line). The chemical structure of ADN is shown as inset. (b) The linear transmission spectra of a 1-cm ADN solution sample of $0.05 \mathrm{M}$ concentration and a $1-\mathrm{cm}$ pure DMSO sample. cence spectra of the solution samples of different concentration and path lengths are also shown in Fig. 2 (nonsolid lines). From Fig. 2 one can see three salient features: (i) the peak fluorescence wavelengths for all measured spectral curves remain nearly the same, (ii) the whole spectral distributions for one- and two-photon induced fluorescence are exactly the same for the same 1-mm solution sample of low $(0.0005 M)$ concentration, and (iii) for the high $(0.05 M)$ concentration sample, the measured two-photon induced fluorescence spectra have been partially cut away in its "blue" tail range. The last feature is due to reabsorption of the fluorescence spectral components which fall in the "red" tail range of the linear absorption band. As demonstrated in Fig. 2, the reabsorption influence is proportional to the propagation distance of the fluorescence signals.

The one- and two-photon induced fluorescence decay curves were measured by a high-speed streak camera (C5680-22 from Hamamatsu). These two measured curves were basically identical and follow single-exponential decay kinetics with a lifetime of $\tau=2.7 \mathrm{~ns}$. Based on the spectral data shown in Fig. 2 and the measured temporal decay data, one may conclude that the one- and two-photon induced fluorescence signals originate from the same emitting statei.e., the lowest level of $S_{1}$ singlet states.

\section{SPECTRA PROPERTIES OF 2PP LASING}

The optical setup for our 2PP lasing study is shown in Fig. 3(a) where the $\sim 775$-nm and $\sim 160$-fs pump laser beam is focused via an $f=15 \mathrm{~cm}$ lens into the center of a 1-cm-path-length quartz cuvette, filled with ADN solution in DMSO of $0.05 M$ concentration. Once the input pump pulse energy is higher than a certain threshold value, a highly directional stimulated emission (or cavityless lasing) beam of blue color can be readily observed in both forward and backward directions. Furthermore, for a fixed pump beam, if the cuvette is slightly turned to a position where the incident

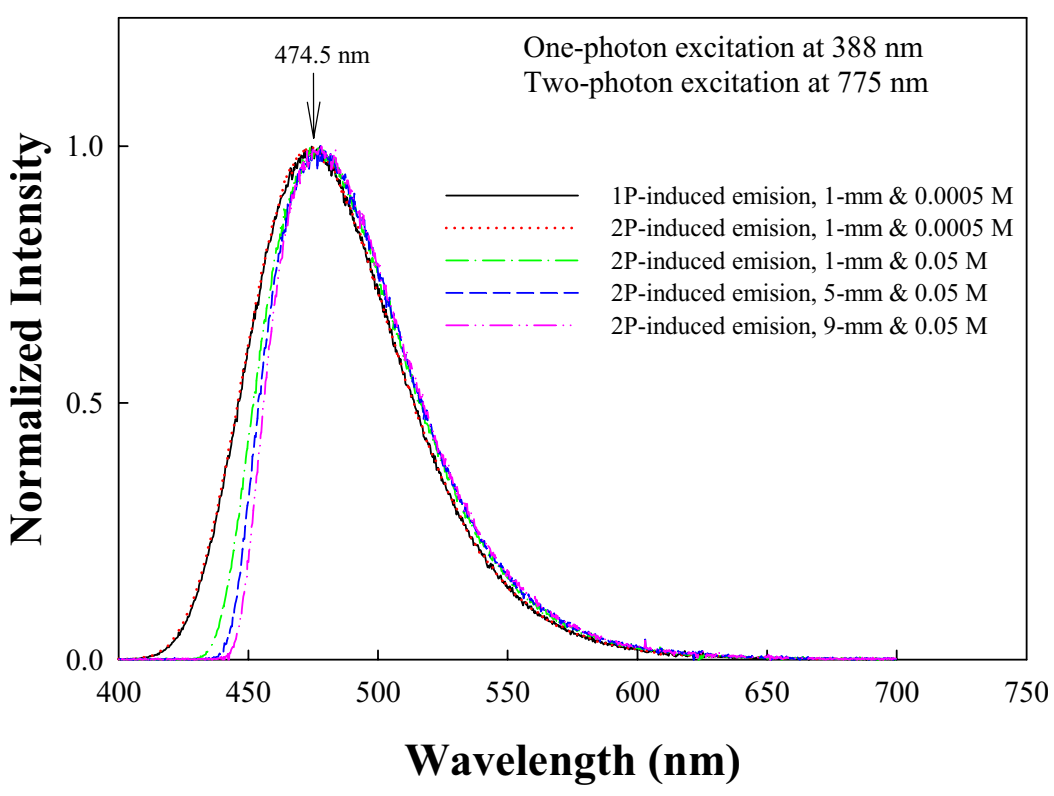

FIG. 2. (Color online) Oneand two-photon induced steadystate fluorescence spectra of ADN in DMSO solution at different concentrations and propagation path lengths of the measured fluorescence signals. 


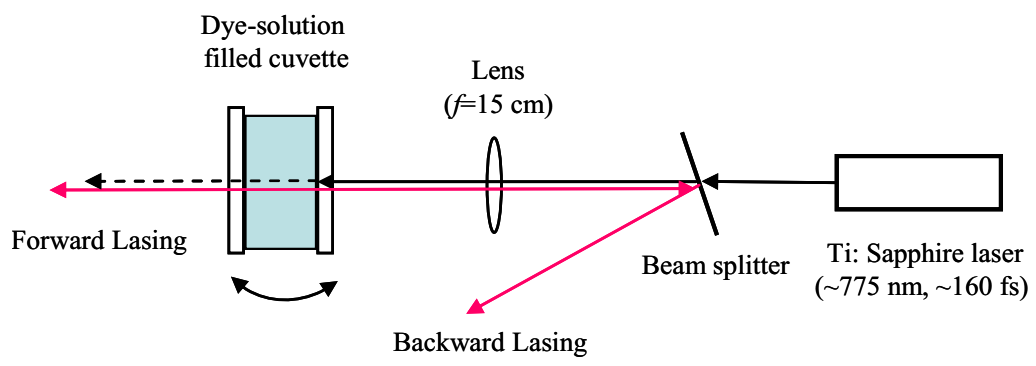

(a)

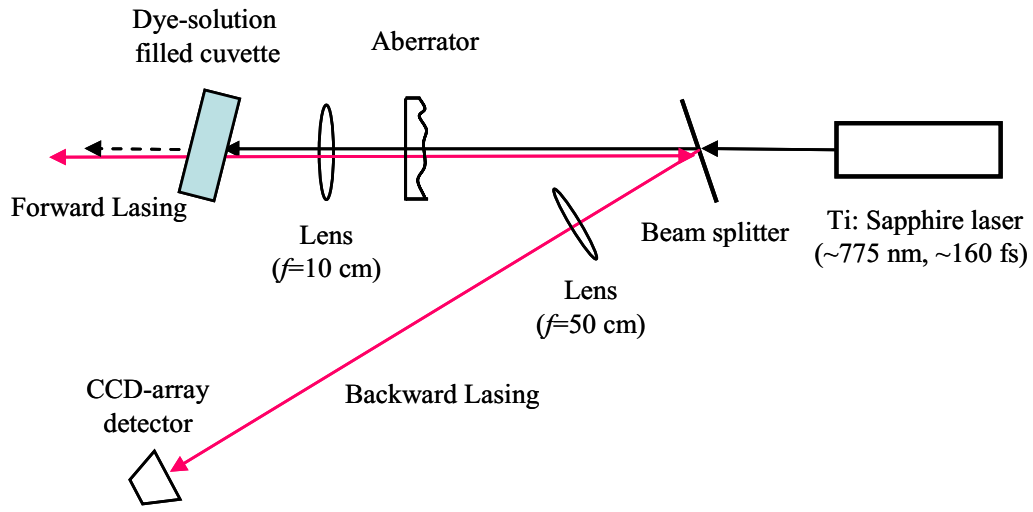

FIG. 3. (Color online) Optical setups for (a) the feedback enhanced lasing experiment and (b) the optical phase-conjugation experiment.

(b)

angle is close to zero degree, a significant enhancement of the output-frequency-upconversion lasing in both directions can be observed. This type of lasing enhancement is similar to what we previously reported by utilizing another gain medium [17], and it is mainly attributed to the reflection of stimulated emission pulses from the glass-air surfaces of the two parallel optical windows of the cuvette. When the incident angle of the pump beam with the windows' normal is larger than $3^{\circ}-5^{\circ}$, no reflection enhancement is observed.

The 2PP lasing output spectral structures were measured by a grating spectrometer (Holo Spec from Kaiser Optical Systems) with a spectral resolution of $\sim 1 \mathrm{~nm}$. The measurements indicated that at a given pump energy level, the forward and backward cavityless lasing output spectra were nearly the same (within our spectral resolution) with or without reflection feedback. On the other hand, the peak lasing wavelength position was slightly dependent on the pump energy. The measured forward lasing spectral curves obtained at three different pump energy levels are shown in Fig. 4; for comparison, the two-photon induced fluorescence spectrum previously measured in the same sample solution is also given in the same figure. From Fig. 4 one can see two interesting features. First, the 2PP lasing peak wavelengths are always blueshifted from the corresponding peak fluorescence wavelength position. At a low pump energy level of $4.6 \mu \mathrm{J}$, the lasing peak wavelength is $466.5 \mathrm{~nm}$, which is $8 \mathrm{~nm}$ blueshifted with respect to the fluorescence peak wavelength $(474.5 \mathrm{~nm})$ position. Second, with increasing the pump energy level, the peak lasing wavelength shifts slightly to a longer-wavelength position. For example, when the pump level increases from 4.6 to $74 \mu \mathrm{J}$, the lasing peak wavelength shifts from 466.5 to $471.5 \mathrm{~nm}$. These two spectral features of 2PP lasing will be further discussed in Sec. V.

\section{DYNAMIC BEHAVIOR OF 2PP LASING}

Shown in Fig. 5 are the measured wave form of the transmitted pump pulse and that of a single forward 2PP lasing pulse at a pump energy level of $\sim 60 \mu \mathrm{J}$ without reflection enhancement. This measured curve was obtained by using the streak camera working in its 0.5 -ns scanning scale. The apparent separation $\delta$ between these two pulses was $\delta$ $\approx 20 \mathrm{ps}$. The separately measured group velocity dispersion delay $\delta^{\prime}$ between the spectral components of $\sim 775 \mathrm{~nm}$ and $\sim 470 \mathrm{~nm}$ through our whole optical system (including the 1-cm-path-length lasing cuvette and the lenses outside and inside the camera) was about $\delta^{\prime} \approx 7-8 \mathrm{ps}$. Therefore, the net delay between the pump pulse and the forward lasing pulse is approximately $\delta^{\prime \prime}=\delta-\delta^{\prime} \approx 12-13$ ps. The physical meaning of this delay is that after this time period, most of the excited ADN molecules finally reach the (fluorescence) emitting level(s) through internal conversion and vibrational relaxation processes [18-20]. It is also noted that this delay is considerably longer than that we observed before in salt-type lasing dye solutions [14].

Figure 6 shows the temporal wave forms of the transmitted pump pulse and three forward lasing pulses at a pump energy level of $\sim 80 \mu \mathrm{J}$ with reflection enhancement. Under this condition, the whole forward lasing output consists of three major pulses separated by $\Delta \approx 60 \mathrm{ps}$ with respect to 


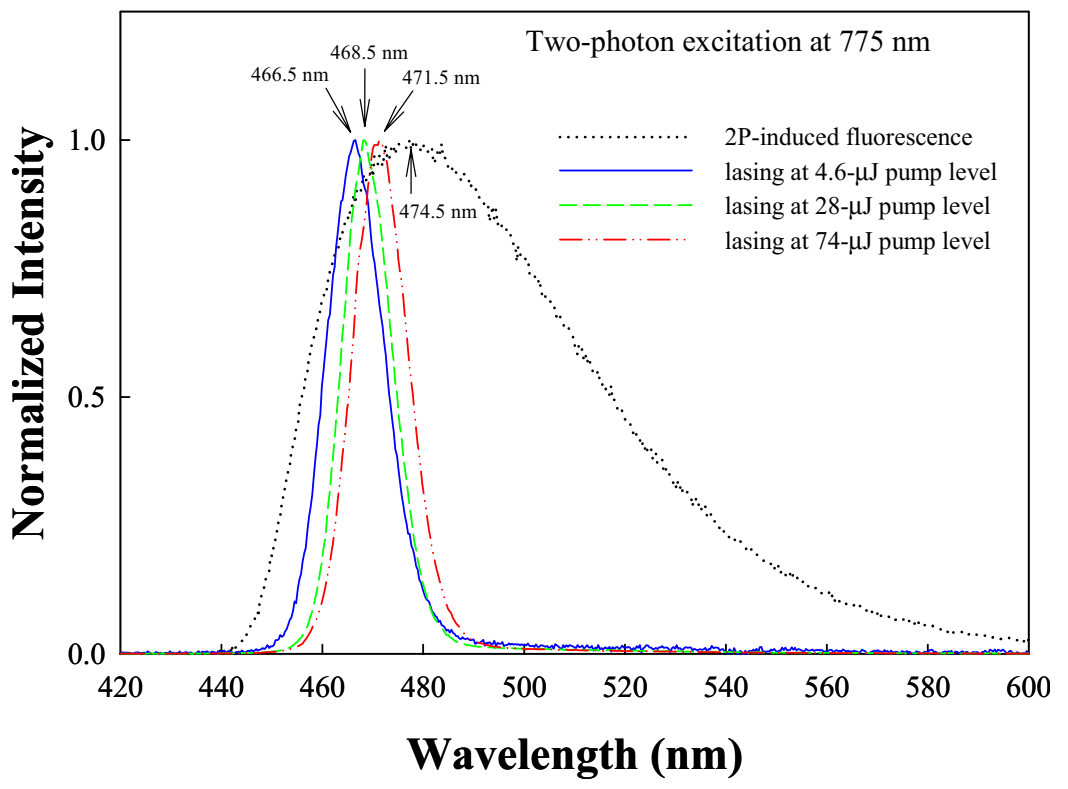

FIG. 4. (Color online) 2PP forward lasing spectra at three different pump energy levels and the corresponding fluorescence spectrum of ADN (0.05M) in DMSO.

each other. Considering the 1-cm optical path length of the cuvette and the thickness of the optical window of $1 \mathrm{~mm}$, the round-trip time of the photons within the cavity formed by the two window-air surfaces is $\sim 120$ ps. The formation of the multipulse structure of the forward lasing output can be clearly explained by means of the inset in Fig. 6, where the pump pulse is focused in the center of the gain medium and both the initial forward and backward stimulated emission signals start from this point. The forward initial stimulated emission is further amplified by passing through half of the effective gain length and finally forms the first lasing pulse that is the same as what we observed in Fig. 5 without feedback enhancement. Similarly, the backward initial stimulated emission experiences the same amplification passing through half of the cuvette's path length and is partially reflected at the glass-air surface of the entrance window. The reflected backward lasing signal will get further amplification by passing through the whole effective gain length and finally form the second output lasing pulse. At the same time, the par- tially reflected first forward lasing pulse at the exit window and sequentially at the entrance window will get a double path-length gain and finally form the third output laser pulse. The above explanation is acceptable, if we assume that the pump pulse generated peak gain (population inversion) can last longer than 150-200 ps.

There is one issue that should be discussed: i.e., why the measured lasing lasting time (150-200 ps) is much shorter than the fluorescence lifetime $(\sim 2.7 \mathrm{~ns})$. In this case, we may consider two factors: one is the dynamic curve of the gain medium, and the second is the threshold requirement for generating lasing. In general, the dynamic gain curve for a gain medium is determined by the population inversion decay curve in time scale, and the latter can be approximately recognized as the fluorescence decay curve without considering the depletion effect. Due to the threshold requirement, only the top section of the exponential decay curve can provide high enough gain over a certain propagation length (effective gain length or focal depth of the pump beam) to over-

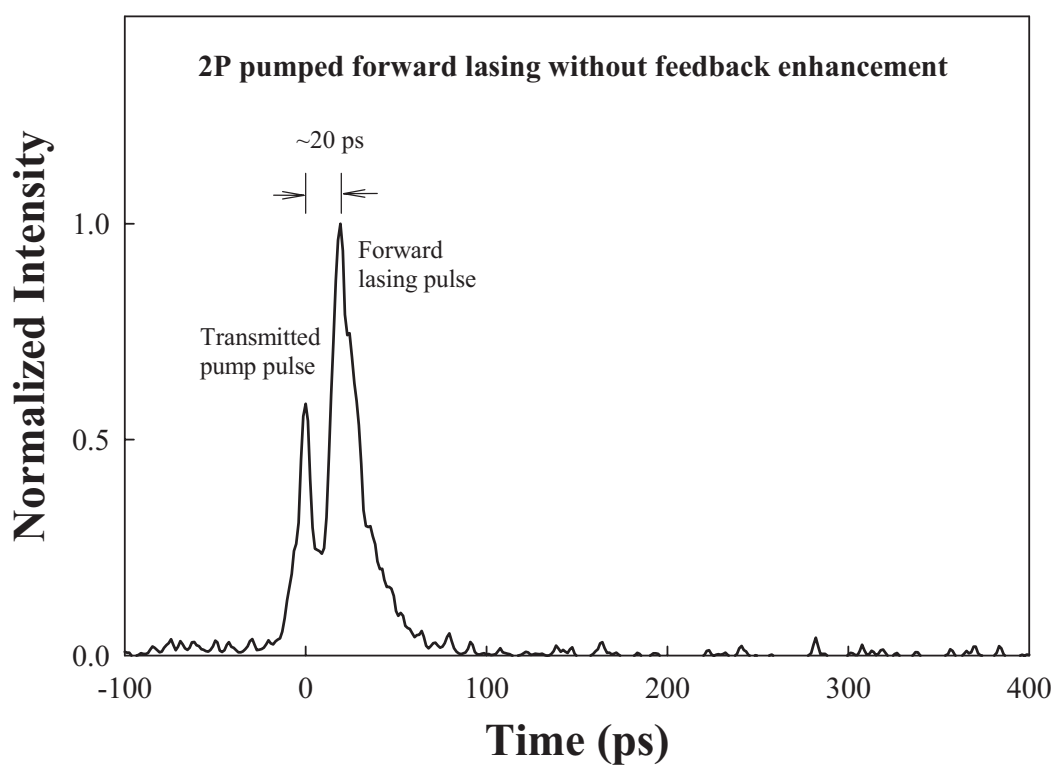

FIG. 5. Temporal wave forms of the transmitted pump pulse and the forward lasing pulse without feedback enhancement. 


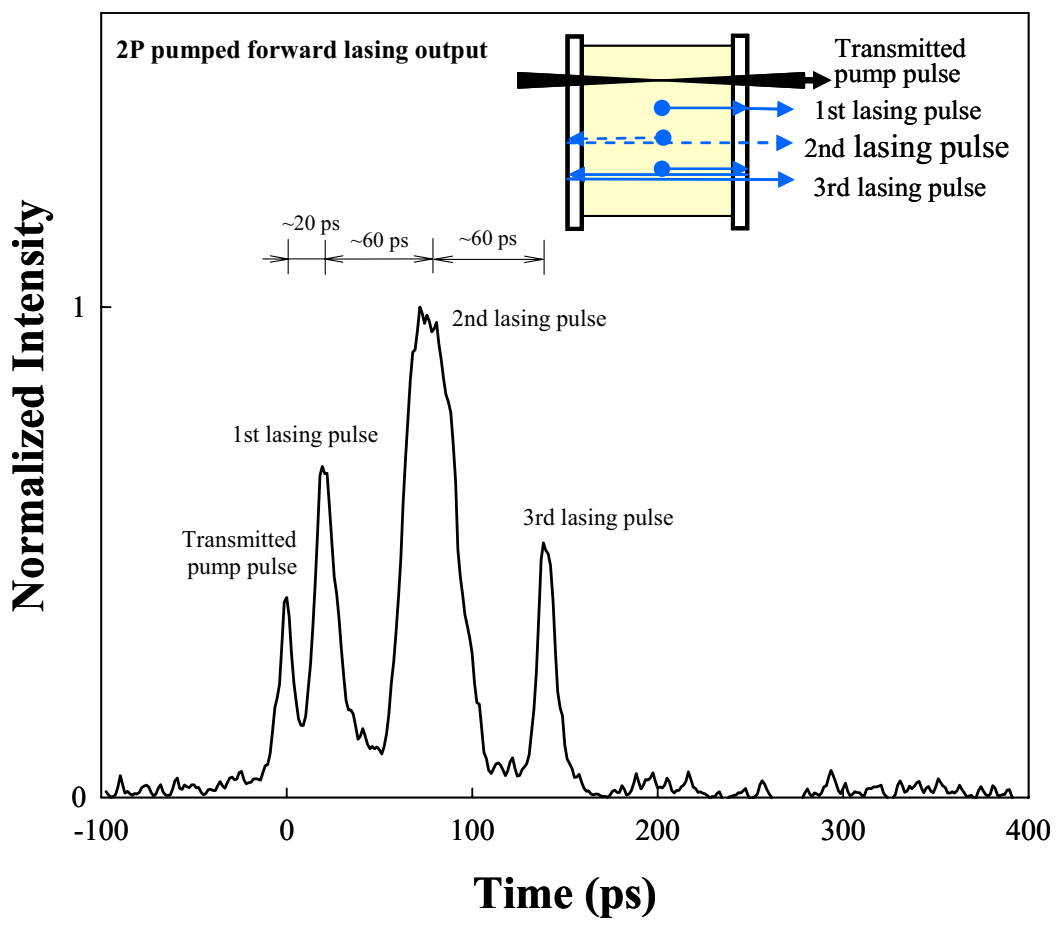

FIG. 6. (Color online) Temporal wave forms of the transmitted pump pulse and the forward lasing pulses with feedback enhancement.

come the losses caused by various attenuation mechanisms. This is the first reason why the lasing time period is much shorter than the fluorescence lifetime of the gain medium under ultrashort laser pump condition. The second reason is that once the lasing is taking place near the top position of the exponential decaying curve of gain or population inversion, the photon simulated amplification will expedite the depletion of population inversion of the gain medium, which leads to further shortening of the effective gain decay in comparison with the normal fluorescence decay curve.

\section{SOME PHYSICAL EXPLANATIONS OF THE LASING SPECTRAL PROPERTIES}

As described in Sec. III and shown by Fig. 4, there are two salient features of 2PP lasing spectral behavior: one is the blueshift of the lasing peak wavelength with respect to the fluorescence peak wavelength; the other is a slight redshift of the lasing peak wavelength with the increase of pump energy. Both of these two features can be qualitatively explained by the so-called solvent relaxation mechanism, which commonly occurs in organic chromophore solutions when a polar solvent (such as DMSO) is involved [18-20]. The influence of solvent relaxation on the transient fluorescence emission spectral structure can be well understood by means of schematic illustration shown in Fig. 7. Without optical excitation, most chromophore molecules stay in their lowest vibrational level of the $S_{0}$ ground electronic state, while each of them exhibits an equilibrium interaction with the surrounding solvent molecules. After applying an ultrashort laser pulse, some chromophore molecules are excited to a vibrational level of higher electronic states and then rapidly relaxed to the lowest vibrational level of the $S_{1}$ singlet state, from which the fluorescence emission can be observed with a relatively long lifetime (usually in the ns regime). For those excited chromophore molecules just reaching the fluorescence emitting level, the pump field induced molecular dipole moment vector of an excited chromophore molecule is changed in magnitude and/or direction, compared to those of nonexcited molecules. In this case, the excited chromophore molecule has a tendency to reorientate the surrounding solvent molecules to keep a minimum free energy of the cage consisting of this excited chromophore molecule and surrounding solvent molecules and to lower their interaction energy. This solvent relaxation may take a time period of several tens to several hundreds of picoseconds depending on specific solutes, solvents, and other conditions (such as temperature).

According to this explanation, at the beginning of the process, the fluorescence emission peak wavelength should be shorter than that at the end of process. Measuring the timeresolved fluorescence spectral structure, one may observe that the peak fluorescence emission wavelength will continuously shift toward the longer-wavelength position during the time period of solvent relaxation. For this purpose, we measured the time-resolved fluorescence spectral change by using the streak camera in conjunction with a grating spectrometer. Shown in Fig. 8(a) is the measured fluorescence spectral distribution integrated in a time period of $250 \mathrm{ps}$ from the starting point of fluorescence emission, whereas shown in Fig. 8(b) is the spectral distribution integrated in a period from 900 to $2500 \mathrm{ps}$. In the former case, the peak emission wavelength is $\sim 469 \mathrm{~nm}$ which is close to the lasing wavelengths, whereas in the latter case, the peak emission wavelength is $\sim 474 \mathrm{~nm}$ that is close to the steady-state fluorescence peak wavelength. Usually, it is assumed that the peak lasing wavelength position is determined by the transient peak fluorescence wavelength position; therefore, it can be explained why the observed lasing wavelengths are shorter than the peak wavelength of the steady-state fluorescence 


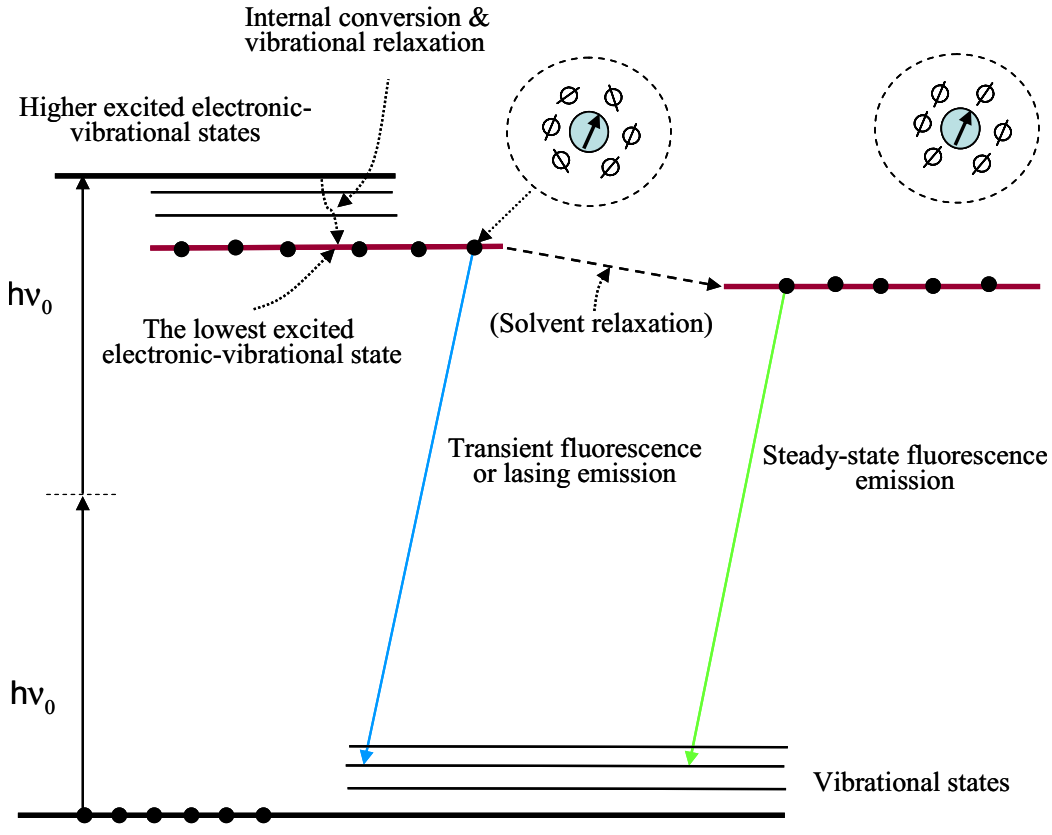

FIG. 7. (Color online) Schematic energy levels and transition pathways of chromophore molecules in a polar solvent.

Electronic ground state

spectrum. It is noted that the measured lasing period in our condition is about 120 ps (see Fig. 6), which must be shorter than the solvent relaxation time.

The same mechanism may also explain why the lasing wavelength increases slightly when the pump energy is increased. In this situation, it is assumed that a larger pump energy value leads to a higher local temperature increase due to 2PA. We measured the fluorescence spectra of the sample solution at different temperature levels from 23 to $80^{\circ} \mathrm{C}$. There was no change in the peak wavelength position of the measured steady-state fluorescence spectra, within the $\sim 1$-nm experimental uncertainty. On the other hand, it may be assumed that the solvent relaxation time of the sample solution is getting shorter when the temperature is getting higher. This speculation is based on the reasoning that the reorientation time of solvent molecules depends on the viscosity of the solvent, when the temperature is getting higher the viscosity is getting lower. At high pump levels, the increase of local temperature in the lasing region may cause the solvent relaxation time to be comparable with the lasing time period. Therefore, the apparent peak wavelength position of the measured (integrated) lasing spectrum should be slightly redshifted, compared to that observed at a low pump energy level, as shown in Fig. 4.

Experiments were conducted to determine the polarization property of the 2PP lasing output. The results show that when the input pump laser beam is nearly linearly polarized, the output lasing beam exhibits a perfect linear polarization.

\section{LASING OUTPUT-INPUT RELATIONSHIP}

The nonlinear transmission of the 1-cm-path-length solution sample of $0.05 \mathrm{M}$ concentration was measured as a function of the input 775-nm laser pulse energy (or peak intensity) under the condition of an incident angle $\geqslant 5^{\circ}$. The results are shown in Fig. 9(a), from which one can see that, when the input pulse energy is higher than 30-50 $\mu \mathrm{J}$, the nonlinear transmission does not decrease with further increase of the input levels. Two possible mechanisms may lead to this nonlinear transmission behavior: one is a selfdefocusing effect and the other is two-photon absorption saturation effect. To reduce the possibility of the thermal defocusing effect, an input 775-nm beam consisting of $1-\mathrm{kHz}$ laser pulses was passed through a rotating chopper with a single opening slot. The rotation speed of the chopper was 40 rounds per second, with an opening/blocking ratio of $1 / 10$. After passing through this chopper, there were 40 groups of laser pulses per second and each group contained only two to three individual laser pulses. Thus the averaged laser induced thermal effect was reduced by a factor of 10 .

The saturation effect of $2 \mathrm{PA}$ in organic chromophore solutions in the fs regime is mainly due to instant depletion of the ground-state population of chromophore molecules. The influence of 2PA saturation on the nonlinear transmission can be described as [21]

$$
T\left(I_{0}\right)=\frac{1}{1+\beta_{0} I_{0} L /\left[1+\left(I_{0} / I_{\mathrm{s}, 2 \mathrm{pa}}\right)^{2}\right]} .
$$

Here $I_{0}$ is the incident laser intensity, $L$ is the optical path length of the sample solution, $\beta_{0}$ is the unsaturated 2PA coefficient of the nonlinear absorbing medium, and $I_{s, 2 \mathrm{pa}}$ is the saturation intensity of the given medium. The best fitting curve of applying Eq. (1) to our experimental results is shown in Fig. 9(a) by a solid-line curve with the fitting parameters of $\beta_{0}=0.0001 \mathrm{~cm} / \mathrm{GW}$ and $I_{s, 2 \mathrm{pa}}=6250 \mathrm{GW} / \mathrm{cm}^{2}$.

The measured overall lasing output pulse energy as a function of the input pump energy under the condition with feedback enhancement is shown in Fig. 9(b). A simple assumption is that the lasing energy $\left(E_{\text {las }}\right)$ is proportional to 


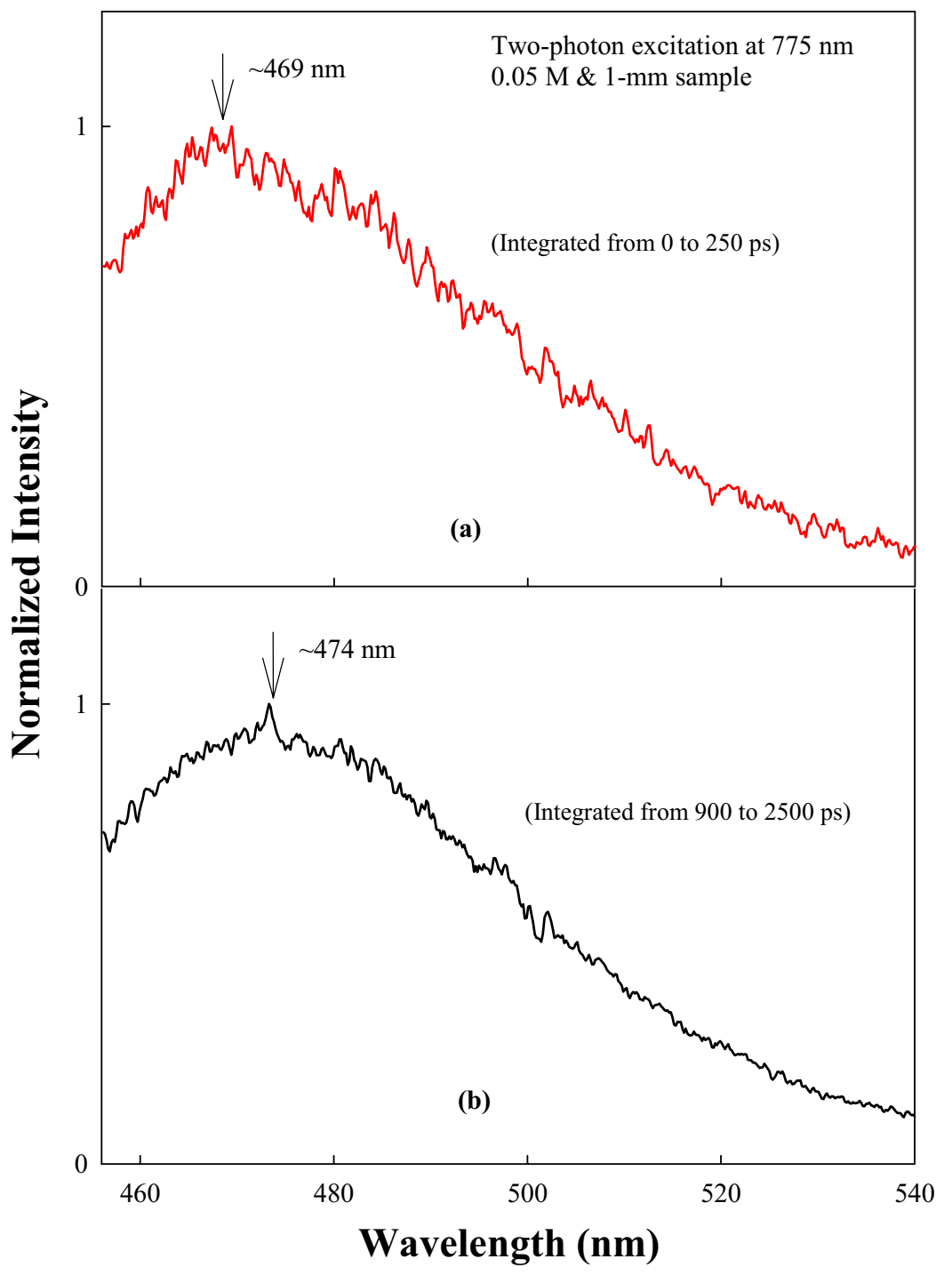

FIG. 8. (Color online) Twophoton induced fluorescence spectra integrated in two different time periods.

that portion of the pump energy $\left(E_{\text {pum }}\right)$ absorbed by the gain medium. Then we have

$$
E_{\text {las }} \propto(1-T) E_{\text {pum }},
$$

where $T$ is nonlinear transmission due to 2PA. Considering the threshold requirement for lasing, the above expression can be further modified as

$$
E_{\text {las }} \approx A(1-T) E_{\mathrm{pum}}-B
$$

Here $A$ and $B$ are two proportional constants. The solid-line curve shown in Fig. 9(b) is the best fit by using the much simplified relationship described by Eq. (3).

From Fig. 9(b) one can see that at the input level of $\sim 100 \mu \mathrm{J}$, the output lasing energy was $1.85 \mu \mathrm{J}$. The overall conversion efficiency from the input pump energy to the output lasing energy was $\eta \approx 2.3 \%$. At this input level, the nonlinear transmission was $\sim 0.73$; therefore, the net conversion efficiency from the absorbed pump energy to the lasing energy should be $\eta^{\prime} \approx \eta /(1-0.73) \approx 8.5 \%$.

\section{OPTICAL PHASE-CONJUGATION PROPERTY OF BACKWARD 2PP LASING}

So far, there are three major approaches to generate optical phase-conjugate waves: degenerate four-wave mixing (FWM) [22-24], backward stimulated scattering [25,26], and backward stimulated emission [27-30]. Among these totally different approaches, there is one thing in common: that is, the formation of intense light-field induced volume holographic grating and the subsequent wave-front reconstruction. For the first approach, the holographic grating in a nonlinear medium is formed by one of the two counterpropagating pump beams with the signal beam, and the resultant backward phase-conjugate beam can be visualized as the diffracted wave from this grating reading by another pump beam [22-24]. For the other two approaches, a volume holographic grating in a gain medium of stimulated scattering (or stimulated emission) can be formed by the input pump beam [26]. In the latter case, according to the original idea of Gabor's principle, after passing through an aberrator plate or a phase-disturbing medium, the input pump laser field can be thought as a superposition of two portions: one is a stronger and undisturbed wave portion with a regular wave 

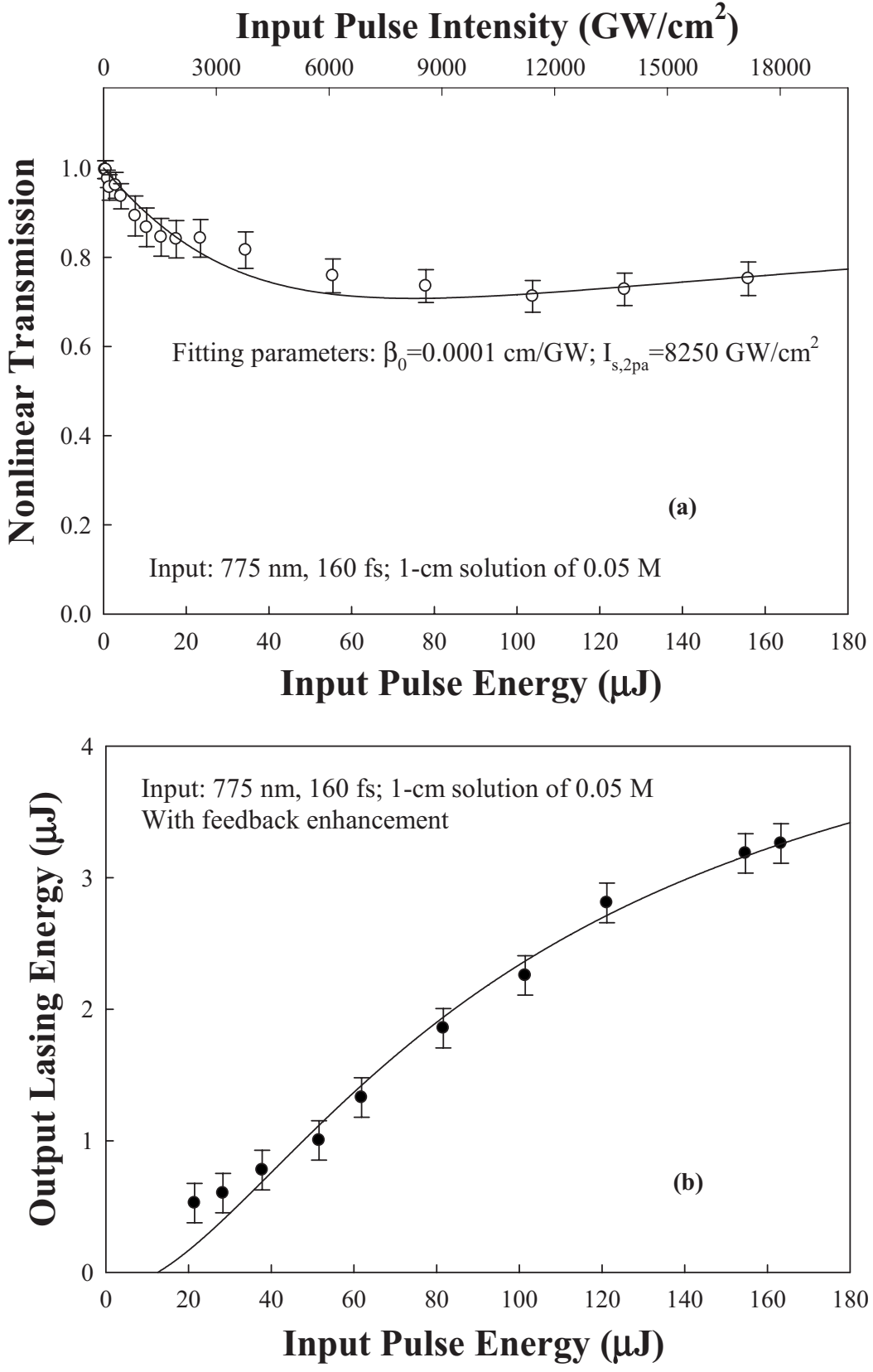

FIG. 9. (a) Measured nonlinear transmission versus the input pulse energy and intensity. (b) Output lasing energy versus the input pump energy. The solid-line curves are the best-fitting curves given by Eqs. (1) and (3), respectively. front, and the other is a weaker and disturbed wave portion carrying aberration influences. These two portions of the pump field interfere with each other and lead to a characteristic three-dimensional and fluctuated intensity distribution inside the gain medium. Since there is a nonlinear refractiveindex change depending on the local pump laser intensity, a holographic phase grating is formed [27].

In two-photon pumped lasing case, once a pump field induced holographic grating has been formed inside the gain medium and the intensity level of the undisturbed pump portion is higher than a certain threshold value, an initial backward stimulated emission wave can be generated at a new frequency with a regular wave front. During the propagation of this backward wave through the grating region, another diffracted wave at the same frequency is created with a wave front resembling the disturbed pump portion. In this sense, it is a nondegenerate and quasicollinear FWM process. The optical phase-conjugation property of the backward 2PP lasing beam has already been proved in the ns regime [28,29]. Now we further report the same type of optical phaseconjugation property of the backward stimulated emission generated by fs-laser pump pulses.

To assess the optical phase conjugation property of the backward stimulated, an optical layout design shown in Fig. 3(b) is employed. In this case, the pump laser beam was focused by an $f=10 \mathrm{~cm}$ lens into the center of the gain cuvette with an incident angle $\geqslant 5^{\circ}$ to avoid the reflection influence from the windows. Between the focusing lens and the beam splitter a removable aberrator was placed. The aberrator was a 1-mm-thick glass slide (model No. 2947 from Corning) corroded in a diluted hydrofluoric acid. The backward stimulated emission output beams was passing through an $f=50 \mathrm{~cm}$ lens and focused on the surface of a chargecoupled-device (CCD) array detector. From the spot size of 

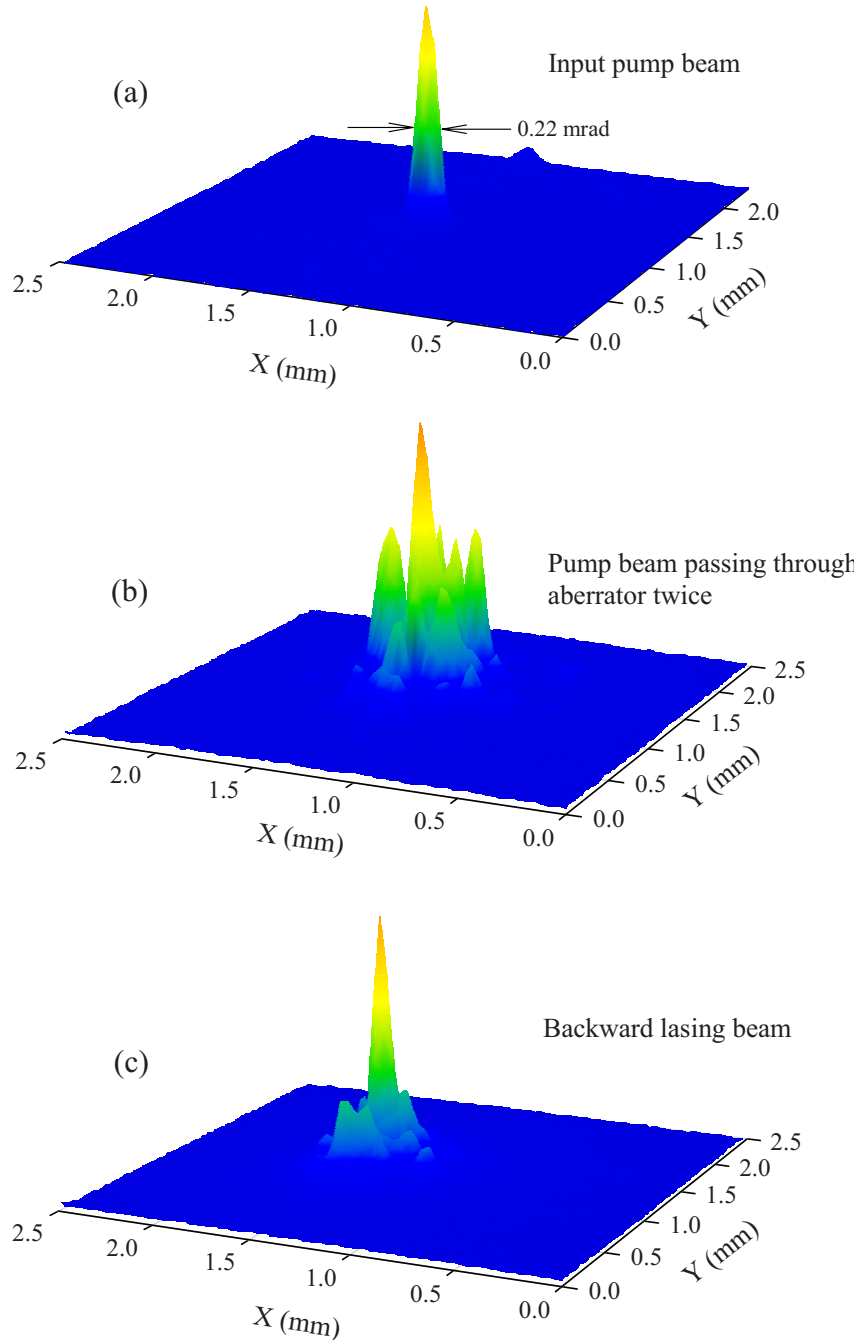

FIG. 10. (Color online) Measured far-field distributions of (a) the input pump beam, (b) the pump beam passed through the aberrator twice, and (c) the backward lasing beam after passing through the aberrator.

the measured far-field pattern, the divergence angle of the beam can be determined.

Shown in Fig. 10(a) is the measured far-field intensity distributions of the input pump beam focused by the same $f=50 \mathrm{~cm}$ lens. The measured divergence angle for the pump beam was $\sim 0.22 \mathrm{mrad}$. Shown in Fig. 10(b) is the far-field pattern of the pump beam after passing through the aberrator twice (one forward pass and, after reflection from a mirror, one backward pass). Under the condition of placing this aberrator, the lasing threshold was significantly increased and a much higher pump level was needed. Shown in Fig. 10(c) is the measured far-field pattern of the backward lasing beam generated at a pump level of $140 \mu \mathrm{J}$, indicating a sharp central peak and only minor aberration influences. Based on the results shown in Fig. 10, one may recognize the optical phase-conjugation property of the backward stimulated emission under our fs-pulse pump conditions.

\section{CONCLUSIONS}

By using $\sim 160$-fs laser pulses at $\sim 775 \mathrm{~nm}$ as the twophoton pump source, blue lasing at a wavelength of $\sim 470 \mathrm{~nm}$ was observed in an organic chromophore (ADN) solution in DMSO. Without feedback from the reflection of the windows of the cuvette, only a single output cavityless lasing pulse was observed, with a duration of $\sim 20 \mathrm{ps,}$ whereas with reflection feedback from the two parallel optical windows, three lasing pulses separated by $\sim 60$ ps spacing were observed in both forward and backward directions. In addition, the backward lasing pulse showed excellent phase-conjugation properties, which was demonstrated by removal of the influence of an aberrator. The lasing peak wavelength was blueshifted compared to the peak fluorescence wavelength. On the other hand, a slight redshift of the lasing peak wavelength was observed with increasing pump energy. These two spectral features of lasing output observed under our experimental conditions were semiquantitatively explained by the transient nature of peak fluorescence (or gain) wavelength position related to the solvent relaxation process.

\section{ACKNOWLEDGMENTS}

This work was supported by the U.S. Air Force Office of Scientific Research. S.J. and N.J.T. thank the National Science Foundation for financial support through Grant No. NSF CHE 04-15516. H.-Y.Q. is supported by the Swedish Foundation for Strategic Research (SSF).
[1] P. Qiu and A. Penzkofer, Appl. Phys. B: Photophys. Laser Chem. 48, 115 (1989).

[2] A. S. Kwok, A. Serpenguzel, W. F. Hsieh, R. K. Chang, and J. B. Gillespie, Opt. Lett. 17, 1435 (1992).

[3] A. Mukherjee, Appl. Phys. Lett. 62, 3423 (1993).

[4] G. S. He, J. D. Bhawalkar, C. F. Zhao, C.-K. Park, and P. N. Prasad, Opt. Lett. 20, 2393 (1995).

[5] G. S. He, L. Yuan, Y. Cui, M. Li, and P. N. Prasad, J. Appl. Phys. 81, 2529 (1996).

[6] G. S. He, L. Yuan, P. N. Prasad, A. Abbotto, A. Facchetti, and G. A. Pagani, Opt. Commun. 140, 49 (1997).

[7] G. S. He, K.-S. Kim, L. Yuan, N. Cheng, and P. N. Prasad,
Appl. Phys. Lett. 71, 1619 (1997).

[8] G. S. He, R. Signorini, and P. N. Prasad, IEEE J. Quantum Electron. 34, 7 (1998).

[9] A. Abbotto, L. Beverina, R. Bozio, S. Bradamante, C. Ferrante, G. A. Pagani, and R. Signorini, Adv. Mater. (Weinheim, Ger.) 12, 1963 (2000).

[10] Y. Ren, Q. Fang, W.-T. Yu, H. Lei, Y.-P. Tian, M.-H. Jiang, Q.-C. Yang, and T. C. W. Mak, J. Mater. Chem. 10, 2025 (2000).

[11] G. S. He, P. P. Markowicz, T.-C. Lin, and P. N. Prasad, Nature (London) 415, 767 (2002).

[12] G. S. He, J. Dai, T.-C. Lin, P. P. Markowicz, and P. N. Prasad, 
Opt. Lett. 28, 983 (2003).

[13] C. Ye, J. Wang, and D. Lo, Appl. Phys. B: Lasers Opt. 78, 539 (2004).

[14] G. S. He, T.-C. Lin, S.-J. Chung, Q. Zheng, C. Lu, Y. Cui, and P. N. Prasad, J. Opt. Soc. Am. B 22, 2219 (2005).

[15] S. Jockusch, Q. Zheng, G. S. He, H. E. Pudavar, D. J. Yee, V. Balsanek, M. Halim, D. Sames, P. N. Prasad, and N. J. Turro, J. Phys. Chem. C 111, 8872 (2007).

[16] D. J. Yee, V. Balsanek, and D. Sames, J. Am. Chem. Soc. 126, 2282 (2004).

[17] G. S. He, Q. Zheng, P. N. Prasad, and J. G. Grote, Opt. Lett. 31, 359 (2006).

[18] (a) G. S. He, C. Lu, Q. Zheng, A. Baev, M. Samoc, and P. N. Prasad, Phys. Rev. A 73, 033815 (2006); (b) V. Kimberg, S. Polyutov, F. Gel'mukhanov, H .Ågren, A. Baev, Q. Zheng, and G. S. He, ibid. 74, 033814 (2006).

[19] J. R. Lakowicz, Principles of Fluorescent Spectroscopy (Kluwer Academic/Plenum, New York, 1999), Chaps. 5 and 6.

[20] B. Valeur, Molecular Fluorescence: Principles and Applica- tions (Wiley-VCH, New York, 2001), Chap. 7.

[21] G. S. He, Q. Zheng, A. Baev, and P. N. Prasad, J. Appl. Phys. 101, 083109 (2007).

[22] A. Yariv, IEEE J. Quantum Electron. 14, 650 (1978).

[23] R. W. Hellwarth, J. Opt. Soc. Am. 67, 1 (1977).

[24] Optical Phase Conjugation, edited by R. A. Fisher (Academic, New York, 1983).

[25] B. Ya. Zel'dovich, N. F. Pilipetsky, and V. V. Shkunov, Principles of Phase Conjugation (Springer-Verlag, Berlin, 1985).

[26] G. S. He and S. H. Liu, Physics of Nonlinear Optics (World Scientific, Singapore, 2000). Chap. 9.

[27] G. S. He, Prog. Quantum Electron. 26, 131 (2002).

[28] G. S. He, Y. Cui, M. Yoshida, and P. N. Prasad, Opt. Lett. 22, 10 (1997).

[29] G. S. He, N. Cheng, P. N. Prasad, D. Liu, and S. H. Liu, J. Opt. Soc. Am. B 15, 1086 (1998).

[30] G. S. He and P. N. Prasad, IEEE J. Quantum Electron. 34, 473 (1998). 